\title{
Changes in cortical oscillations linked to multisensory modulation of nociception
}

\author{
Flavia Mancini, ${ }^{1}$ Matthew R. Longo, ${ }^{2}$ Elisa Canzoneri, ${ }^{1,3}$ Giuseppe Vallar ${ }^{4,5}$ and Patrick Haggard ${ }^{1}$ \\ ${ }^{1}$ Institute of Cognitive Neuroscience, University College London, London, UK \\ ${ }^{2}$ Department of Psychological Sciences, Birkbeck College, University of London, London, UK \\ ${ }^{3} \mathrm{CsrNC}$, Centre for Studies and Research in Cognitive Neuroscience, University of Bologna, Cesena, Italy \\ ${ }^{4}$ Department of Psychology, University of Milano-Bicocca, Milano, Italy \\ ${ }^{5}$ Neuropsychological Laboratory, IRCCS Italian Auxological Institute, Milano, Italy
}

Keywords: body representation, electroencephalography, human, multisensory integration, pain, somatosensory

\begin{abstract}
Pain can be modulated by several contextual factors. For example, simply viewing one's own body can reduce pain, suggesting that the visual context may influence the processing of nociceptive stimuli. We studied changes in electroencephalographic (EEG) oscillatory activity related to visual modulation of nociception, comparing cortical oscillations during innocuous or noxious contact heat, while participants viewed either their own hand or a neutral object at the same location. Viewing the body compared with viewing the object reduced the intensity ratings of noxious stimuli, but not of innocuous heat. Time-frequency analysis of EEG data revealed that noxious, as opposed to warm, stimulation was associated with reduced beta $(15-25 \mathrm{~Hz})$ power. Classically, such decreases in oscillatory power indicate increases in sensory cortical activation. These event-related oscillatory changes were moreover modulated by the visual context; viewing one's own body increased noxious stimulation-induced beta oscillatory activity bilaterally, relative to viewing a neutral object, possibly indicating inhibition of cortical nociceptive processing. These results demonstrate that visual-nociceptive interactions involve changes in sensorimotor EEG rhythms.
\end{abstract}

\section{Introduction}

Pain is a complex subjective experience, strongly dependent on contextual factors, such as attention, expectancy, and motivation (Wiech et al., 2008). Multisensory inputs also modulate pain (Legrain et al., 2012). For example, simply looking at one's own body relative to an object can reduce pain levels (Mancini et al., 2011, 2012), and the N2/P2 complex of laser-evoked potentials (Longo et al., 2009). The neural basis of this multisensory modulation is not fully understood.

Interactions between visual and somatosensory brain areas could mediate the modulatory effect of viewing the body on nociception. A recent functional magnetic resonance imaging experiment (Longo et al., 2012) reported reduced laser-induced activity in the ipsilateral primary somatosensory cortex and contralateral operculoinsular cortex during the visual context of seeing the body. The study also showed increased functional coupling between visual and parietal areas that are activated by the visual perception of one's own body, including the 'extrastriate body area' in the lateral occipital cortex (Downing et al., 2001), and areas activated by painful laser stimulation.

In the present study, we investigated whether synchronization of neuronal populations may underlie such visual-nociceptive interac-

Correspondence: Flavia Mancini, as above.

E-mail: f.mancini@ucl.ac.uk

Received 17 September 2012, revised 18 October 2012, accepted 5 November 2012 tions, by studying visual modulation of induced sensorimotor electroencephalographic (EEG) oscillations. Event-related desynchronization and synchronization are generated by reciprocal coupling between excitatory and inhibitory neurons (Hari \& Salmelin, 1997), and are thought to reflect periods of high vs. low cortical activation or cortical excitability respectively (Neuper et al., 2006). Specifically, nociceptive processing is often associated with suppression of alpha and beta rhythms in the contralateral somatosensory cortex, indicating early activation of these regions by ascending nociceptive signals (e.g. Crone et al., 1998; Mouraux et al., 2003; Ploner et al., 2006b). Multisensory interactions can also be reflected in changes of local and long-range neural oscillations. For example, neuronal synchronization in the beta band is also associated with crossmodal processing, particularly in paradigms of passive stimulation (for a review, see Senkowski et al., 2008).

Here, we investigated the effect of multisensory modulation on nociceptive-induced EEG oscillations. We hypothesized that viewing the body would reduce or inhibit the response to nociceptive stimuli in somatosensory areas. We therefore conducted two EEG experiments comparing the perception of innocuous or noxious contact heat in two visual contexts - during vision of one's own hand or of a neutral object at the same spatial location. In Experiment 1, slow ramps of thermal stimulation were administered unpredictably in an event-related design. To preview our findings, viewing the hand relatively to viewing the object increased stimulus-induced alpha and beta oscillations for both noxious and innocuous thermal stimuli. 
However, the unpredictable nature of the stimulation in this experiment did not allow us to distinguish whether this visual effect on electroencephalography was related to nociceptive processing or to pain expectation. Therefore, in Experiment 2, innocuous warmth and noxious heat were presented in a blocked design, so that participants knew in advance which level of thermal stimulation would be used throughout the block. In this case, increased beta power was found selectively for noxious stimuli.

\section{Experiment 1}

\section{Materials and methods}

\section{Participants}

Ten healthy right-handed individuals (mean age 25 years, range 1932 years) participated for payment. Informed consent was obtained from all participants. The study was approved by the University College London ethics committee and conducted in accordance with the principles of the Declaration of Helsinki.

\section{Stimuli and procedure}

We used the mirror box technique (Ramachandran et al., 1995) to induce the visual illusion that the participant's right hand, reflected in a mirror aligned with their sagittal plane, was actually their stimulated left hand. This technique was used in order to keep gaze direction and spatial attention constant between the two visual conditions. A fake thermode probe was applied to the right hand simultaneously, to avoid possible perceptual conflict. Similar visual modulation of somatosensation has previously been shown to occur either when gazing directly at the stimulated hand, or when the mirror box is used (e.g. Kennett et al., 2001; Longo et al., 2008). Thus, the present set-up can be taken as equivalent to participants' direct vision of their left hand. A questionnaire administered after the experiment confirmed that participants felt that they saw their left hand in this condition (Longo et al., 2009) and therefore that the illusion provided by the mirror was successful.

Participants sat at a table, with the left hand behind the mirror and the right hand in front, gazing towards their left hand (see Fig. 1A). The tips of the index fingers were $20 \mathrm{~cm}$ from the mirror. Two visual conditions, 'hand-view' and 'object-view', were presented in different blocks in an ABBA order (initial condition counterbalanced across participants). In the 'hand-view' condition, participants looked into the mirror towards their left hand, and saw the reflection of their right hand. In the 'object-view' condition, the right hand was occluded by a box, and participants saw the reflection of an approximately hand-sized wooden block placed on top of it (approximately $3 \mathrm{~cm}$ above the hand).

Thermal stimulation was delivered to the fingertip of the index or middle finger of the left hand by a $13 \mathrm{~mm}$ diameter Peltier-type thermode (NTE-2A; Physitemp Instruments Inc.). A mechanical arm held the probe in position throughout the experiment, at constant contact pressure. On each trial, the probe temperature was initially

\section{A Experimental procedure}

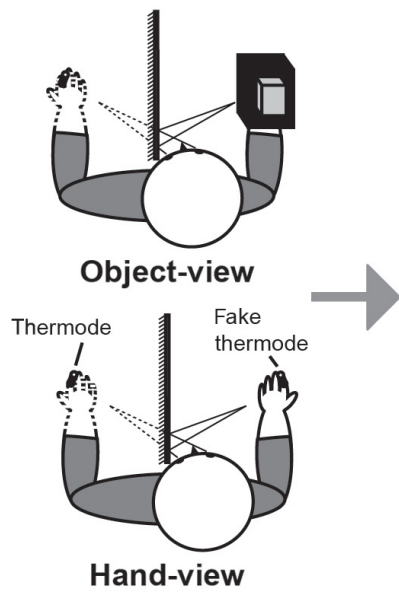

B Behavioural results

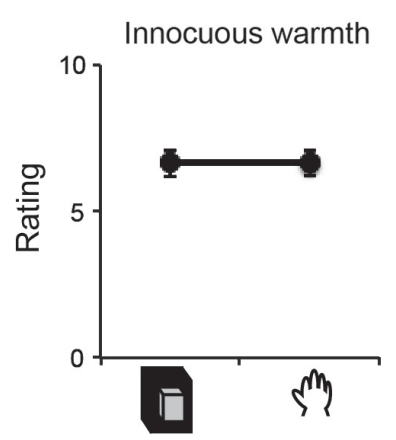

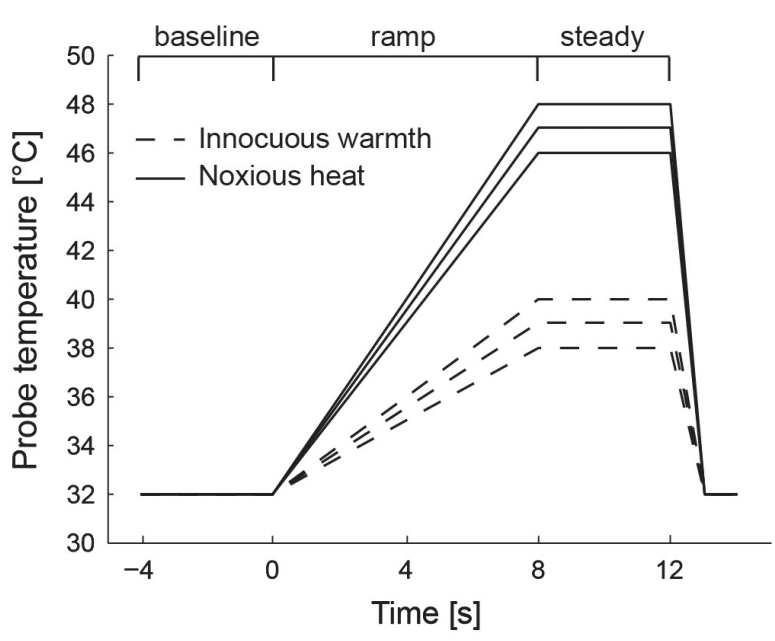

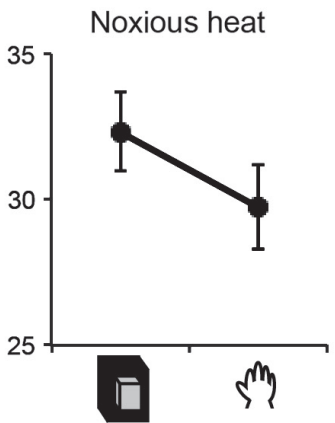

FIG. 1. (A) Procedure of Experiment 1. (B) Behavioral results of Experiment 1; mean intensity ratings \pm SEM, by stimulus intensity and visual context. 
set at normal skin temperature $\left(32{ }^{\circ} \mathrm{C}\right)$, maintained for $3-5 \mathrm{~s}$. During this baseline period, participants continuously looked at either their hand or the object. The temperature was then gradually increased during an $8 \mathrm{~s}$ ramp to unpredictably reach a final stimulus temperature, either in the innocuous warmth $\left(38,39,40{ }^{\circ} \mathrm{C}\right)$ or in the noxious heat $\left(46,47,48{ }^{\circ} \mathrm{C}\right)$ range, in randomized order. These temperatures were chosen as appropriate to stimulate $\mathrm{C}$ - (warmth range) and A-delta fibers (pain range) in the glabrous skin of the hand (Gybels et al., 1979). The final temperature was then maintained at a steady level for $4 \mathrm{~s}$, until a beep marked the end of the trial (Fig. 1A). Vision of the hand/object was maintained throughout the trial. Each condition was repeated 40 times.

The skin temperature was measured by an infrared thermometer at the beginning of $10 \%$ of the trials selected at random, to check that it remained stable across the session (average SD across trials $1.1{ }^{\circ} \mathrm{C}$, SD across subjects $0.4{ }^{\circ} \mathrm{C}$ ).

\section{Electrophysiological recordings}

A SynAmps 2 amplifier system and sCAN 4.3 software (Neuroscan, El Paso, TX, USA) were used to record EEG data. Twenty-six scalp electrodes were recorded (FP1, FP2, F3, Fz, F4, T7, C5, C3, Cz, C4, C6, T8, CP5, CP3, CPz, CP4, CP6, P7, P5, P3, Pz, P4, P6, P8, $\mathrm{O} 1$ and $\mathrm{O} 2)$, according to the 10-20 International EEG System. The online reference electrode was $\mathrm{AFz}$ and the ground electrode was placed on the chin. Electrode impedances were kept below $5 \mathrm{~K} \Omega$. The left and right mastoids were also recorded and used for offline re-referencing. The horizontal electroculogram was recorded from bipolar electrodes placed on the outer canthi of each eye, and the vertical electroculogram was recorded from bipolar electrodes placed above and below the right eye. EEG signals were amplified and digitized at $500 \mathrm{~Hz}$

\section{Electroencephalographic analyses}

The EEG data were preprocessed with EEGLAB (http://sccn.ucsd. edu/eeglab/) (Delorme \& Makeig, 2004). Data were downsampled to $250 \mathrm{~Hz}$, and re-referenced to the average of all of the sensors. Epochs of $14 \mathrm{~s}$ were extracted from the raw EEG data from $2 \mathrm{~s}$ before to $12 \mathrm{~ms}$ after the onset of the ramp. Epochs containing stereotyped artifacts were corrected using blind source separation with independent component analysis (Jung et al., 2000). Further epochs (mean $\pm \mathrm{SD}, 5.97 \pm 6.48 \%$ ) were rejected by visual inspection, on the grounds of eyeblinks and movements.

The EEG oscillations were quantified using sPM8 (http://www. fil.ion.ucl.ac.uk/spm/software/spm8/). A complex Morlet wavelet decomposition of the EEG signal with seven wavelet cycles using a variable time window length was performed across a $2-30 \mathrm{~Hz}$ frequency range, in steps of $1 \mathrm{~Hz}$. The wavelet decomposition was performed for each trial, sensor, and participant. The power at each frequency was logarithmically rescaled ( $\log \mathrm{R}$ in SPM8) for a baseline period defined as the $2 \mathrm{~s}$ immediately before the onset of the stimulus ramp. For analyses of the EEG activity during the visual baseline period (vision of the hand/object before the start of the thermal ramp), absolute, uncorrected, power was used. Time-frequency data were averaged across trials of the same task type to produce an average time-frequency map for each sensor and for each condition.

Given that the purpose of this study was to investigate modulations of somatosensory alpha and beta oscillations (Crone et al., 1998; Mouraux et al., 2003; Raij et al., 2004; Hauck et al., 2007; Stancak et al., 2007), time-frequency data at two electrodes of inter- est (C3 and C4) were averaged across two frequency bands of particular interest, alpha $(8-13 \mathrm{~Hz})$ and beta $(15-25 \mathrm{~Hz})$, for each participant. Factorial ANOVA was performed on both the absolute and baseline-corrected alpha and beta power, comparing the effects of visual context (hand, object), stimulus intensity (innocuous warmth, noxious heat) and hemisphere (contralateral, C4; ipsilateral, C3). The event-related design of our experiment allowed us to independently investigate two different effects on electroencephalography, i. e. the effect of viewing the hand or object, and the effect of noxious or innocuous thermal stimulation.

\section{Results}

\section{Pain intensity ratings}

The ANOVA on intensity ratings revealed significant main effects of visual context $\left(F_{1,9}=5.80, \quad P=0.039\right)$ and stimulus intensity (warmth vs. noxious heat, $F_{1,9}=47.07, P<0.0001$ ), as well as an interaction of these factors $\left(F_{1,9}=5.90, P=0.038\right)$. Viewing the hand significantly reduced the subjective intensity ratings for noxious stimuli $\left(t_{9}=2.58, P=0.032\right)$, as compared with viewing a neutral object (Fig. 1B). In contrast, visual context did not influence the perceived intensity of innocuous warm stimuli $\left(t_{9}=0.03\right.$, $P=0.979$ ). These results replicated previous findings of visuallyinduced analgesia (Longo et al., 2009, 2012; Mancini et al., 2011).

\section{Electroencephalographic oscillations during vision}

We investigated the effect of visual context on spontaneous oscillations during the $2 \mathrm{~s}$ prestimulus baseline period, using a $2 \times 2$ ANOVA on the absolute power with factors of hemisphere and visual context.

In the alpha band, viewing the hand reduced absolute spectral power $\left(F_{1,9}=10.45, P=0.010\right)$, relative to viewing the object. The main effect of hemisphere and the interaction between hemisphere and context were not significant (both $F<1$ ).

The beta absolute power was similarly modulated by the visual context $\left(F_{1,9}=6.85, P=0.028\right)$. As in the alpha band, the main effect of hemisphere and the interaction context by hemisphere were not significant (both $F<1$ ). This indicates that viewing the body relatively to an object suppresses bilaterally alpha and beta oscillations over somatosensory areas.

Stimulus-induced electroencephalographic oscillations - alpha band (8-13 Hz)

These analyses used measures of relative EEG power during the steady phase of innocuous/noxious stimulation, with baseline correction for the visual prestimulus period. An ANOVA with factors of visual context, hemisphere and stimulus intensity was applied.

Viewing the hand increased stimulus-induced alpha-band power (mean \pm SEM, $1.65 \pm 0.41 \mathrm{~dB}$ ) compared with the object-view condition (mean \pm SEM, $0.42 \pm 0.32 \mathrm{~dB}$; visual context: $\left.F_{1,9}=33.24, P<0.0001\right)$. The alpha power was lower for noxious compared with innocuous stimuli (stimulus intensity: $F_{1,9}=11.86$, $P=0.007)$, suggesting an inverse relation between sensory intensity and EEG oscillations. The main effect of hemisphere did not reach the level of significance $\left(F_{1,9}=3.92, P=0.079\right)$. Finally, no interaction was significant (hemisphere by context: $F_{1,9}=2.03$, $P=0.188$; hemisphere by intensity, context by intensity: $F<1$; hemisphere by context by intensity: $F_{1,9}=2.43, P=0.153$ ), indicating that the effects of stimulus intensity and visual context were independent and bilateral (Fig. 2). 

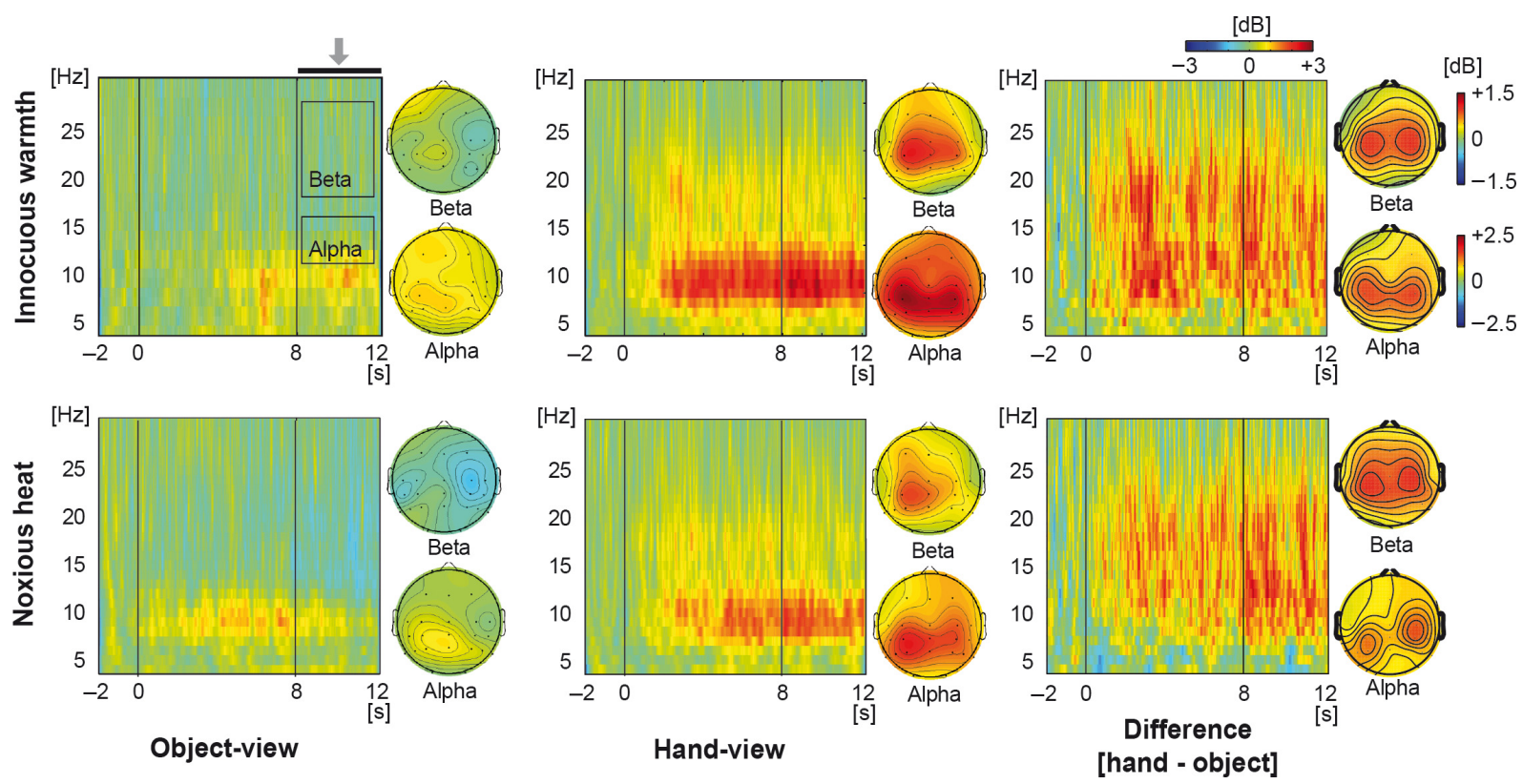

FIG. 2. Experiment 1. Grand mean time-frequency representations of EEG spectral power over the contralateral centro-parietal cortex (electrode C4). Baselinerescaled responses were averaged across 10 subjects. Topographical maps represent average oscillatory power during the steady period $(8-12 \mathrm{~s}$ after the ramp onset) in the alpha $(8-13 \mathrm{~Hz})$ and beta $(15-25 \mathrm{~Hz})$ bands.

\section{Stimulus-induced electroencephalographic oscillations - beta band $(15-25 \mathrm{~Hz})$}

Viewing the hand (mean \pm SEM, $0.68 \pm 0.14 \mathrm{~dB}$ ) increased beta oscillations compared with viewing the object (mean \pm SEM, $-0.28 \pm 0.14 \mathrm{~dB}$; visual context: $\left.F_{1,9}=36.85, P<0.0001\right)$. As in the alpha band, noxious heat reduced beta oscillations, relative to innocuous warmth (stimulus intensity: $F_{1,9}=10.67 P=0.010$ ). The main effect of hemisphere did not reach the level of significance $\left(F_{1,9}=4.15, P=0.072\right)$, and there were no significant interactions (all $F<1$; see Fig. 2).

\section{Discussion}

Experiment 1 yielded three main findings regarding the multisensory modulation of EEG rhythms over the centro-parietal cortex. (i) Viewing the body modulated spontaneous oscillations, reducing absolute power in the alpha and beta band, during the baseline period before thermal stimulation; (ii) noxious heat reduced both alpha and beta spectral power in comparison to innocuous warmth, possibly reflecting increased bilateral activation of the centro-parietal cortex with more intense thermal stimulation; and (iii) thermally-induced alpha and beta power were relatively enhanced, during vision of one's own hand in comparison to a neutral object.

Experiment 1 shows modulations of alpha and beta oscillations that were selective for the visual condition of viewing the body, but were not selective for the intensity of the thermal stimulation. In contrast, the reduction of pain ratings was specific for noxious stimuli. In the event-related design of Experiment 1, the occurrence of pain at the end of the thermal ramp was unpredictable. For this reason, it is possible that EEG modulation for innocuous stimuli might also be introduced by expecting pain that could develop if the ramp were to be continued.

Therefore, in a second experiment we investigated whether the effect of viewing the body on EEG oscillations induced by innocu- ous stimuli reflected modulation of thermal processing, or pain expectation.

\section{Experiment 2}

In Experiment 2, the level of thermal stimulation remained constant throughout a block, and participants were informed of the stimulation level by a cue at the beginning of the block. In contrast, the visual context was now randomized across trials. Each trial was cued for the presence of innocuous or noxious heat, and consisted of three periods - a dark period, unpredictable visual presentation of the hand/object, and visual context plus cued thermal stimulation. This design was chosen in order to provide separate estimates of the EEG effects of thermal stimulus expectation, vision of the hand/ object, and thermal stimulus-induced activity.

\section{Materials and methods}

\section{Participants}

Eleven healthy right-handed individuals participated in Experiment 2 (mean age 23 years, range 19-30 years) for payment.

\section{Stimuli and procedure}

Participants sat at a table, gazing towards their left hand that was placed behind a two-way mirror (see Fig. 3A). The tips of the index fingers were $20 \mathrm{~cm}$ away from the mirror. Innocuous and noxious thermal stimuli were presented in separate alternated blocks, with the initial condition counterbalanced across participants. At the beginning of each block, participants were verbally informed whether the stimulus temperature for that block would be warm or hot. During every trial, computer-controlled LED lights projecting either the left or the right side of the two-way mirror allowed vision of the left hand, or of a hand-sized wooden block, respectively. The timeline of the trial is presented in Fig. 3A; after $2 \mathrm{~s}$ of darkness, 
A Experimental procedure

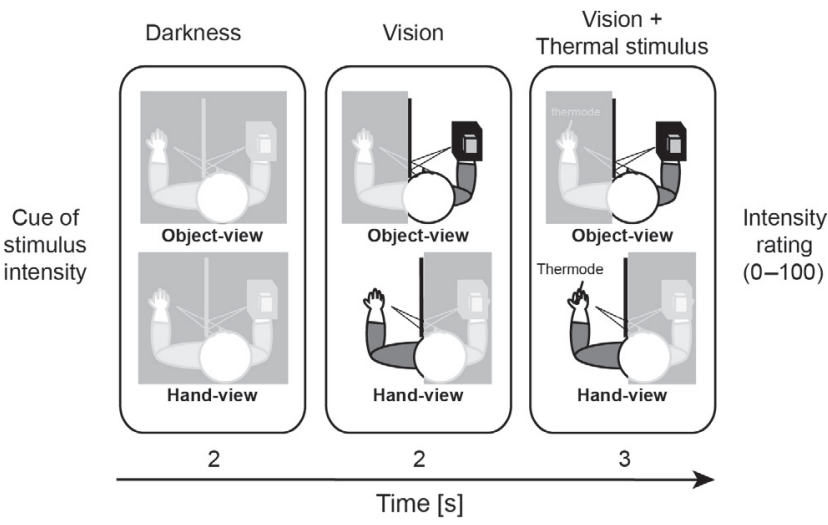

B Behavioural results

Innocuous warm

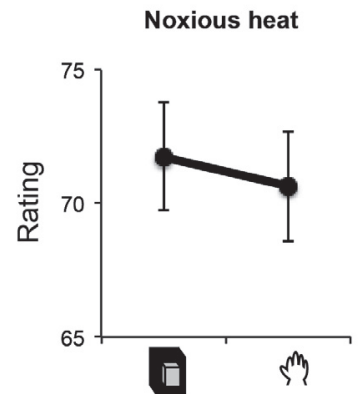

FIG. 3. (A) Procedure of Experiment 2. (B) Behavioral results of Experiment 2 ; mean intensity ratings \pm SEM, by stimulus intensity and visual context.

the light was switched on and participants looked for $2 \mathrm{~s}$ at either the left hand or the reflection of the object onto the mirror. The probe was then applied to the left hand dorsum. Both the probe and the lights were maintained on for a further $3 \mathrm{~s}$. After that time, the lights were switched off, the probe removed, and the subjects were asked to rate the intensity of the stimulation from 0 (no sensation) to 100 (worst pain imaginable).

Whereas in Experiment 1 the probe was in contact with the hand throughout the entire trial, and the temperature was adjusted by a slow increase, in Experiment 2 the participants saw the thermode approaching the hand, and the temperature was maintained constant throughout the contact with the skin. The temperatures selected were the same as in the steady phase of Experiment 1.

The two visual contexts were presented in random order and were never cued. Thirty trials were administered per condition. A block of practice was given at the beginning of the experiment, to familiarize participants with the intensity of the stimulation, and was discarded from statistical analyses. The skin temperature was measured by an infrared thermometer at the beginning of $10 \%$ of the trials, selected at random, to check that it remained stable across the session (average SD across trials $0.5{ }^{\circ} \mathrm{C}, \mathrm{SD}$ across subjects $\left.0.3^{\circ} \mathrm{C}\right)$.

\section{Electroencephalography}

The EEG recordings and analyses were equivalent to Experiment 1, except for the fact that 19 scalp electrodes were used (FP1, FP2, F3, F4, T7, C3, Cz, C4, T8, CP3, CPz, CP4, P7, P3, Pz, P4, P8, O1 and $\mathrm{O} 2$ ). Epochs containing stereotyped artifacts were corrected using blind source separation with independent component analysis
(Jung et al., 2000). Further epochs (mean \pm SD, $7.34 \pm 2.46 \%$ ) were rejected by visual inspection, on the grounds of eyeblinks and movements. Morlet wavelet decomposition of the EEG signal was performed with seven wavelet cycles across a $2-30 \mathrm{~Hz}$ frequency range in steps of $0.5 \mathrm{~Hz}$. Two epochs were extracted from each trial: (i) the last $1 \mathrm{~s}$ of darkness, followed by $2 \mathrm{~s}$ of vision of hand/ object; and (ii) the last $1 \mathrm{~s}$ of vision (hand/object-view), followed by $3 \mathrm{~s}$ of visual plus thermal stimulation.

The alpha $(8-13 \mathrm{~Hz})$ and beta $(15-25 \mathrm{~Hz})$ power extracted from C3 and C4 channels was analysed in order to separately investigate the effect of the following:

1. Stimulus expectancy during darkness. The absolute power during $1 \mathrm{~s}$ of darkness in epoch no. 1 was submitted to $2 \times 2$ ANOvas with factors of hemisphere (C3, C4) and blocked thermal stimulation level (innocuous, noxious stimulation).

2. Visual context relative to darkness. The power during vision $(0.5$ $-2 \mathrm{~s}$ since the onset of the visual event) from epoch no. 1 was logarithmically rescaled to the darkness period (1 s), and submitted to $2 \times 2 \times 2$ ANOVAs with factors of hemisphere, visual context, and intensity cue.

3. Visual plus thermal stimulation relative to vision. The power during $3 \mathrm{~s}$ of visual plus thermal stimulation from epoch no. 2 was logarithmically rescaled for the visual period (last $1 \mathrm{~s}$ of vision), and submitted to $2 \times 2 \times 2$ ANOvas with factors of hemisphere, visual context, and stimulus intensity (innocuous warmth, noxious heat).

\section{Results}

\section{Pain intensity ratings}

The ANOVA on intensity ratings revealed a significant main effect of stimulus intensity (warmth vs. noxious heat, $F_{1,10}=75.18$, $P<0.0001)$, and no main effect of visual context $\left(F_{1,10}=2.22\right.$, $P=0.167)$. The interaction between the stimulus intensity and the visual context was significant $\left(F_{1,10}=8.46, P=0.016\right)$. As in Experiment 1 , viewing the hand significantly reduced the subjective intensity ratings for noxious stimuli $\left(t_{10}=-3.27, P=0.008\right)$ compared with viewing a neutral object (Fig. 3B). In contrast, visual context did not influence the perceived intensity of innocuous warm stimuli $\left(t_{10}=1.21, P=0.255\right)$.

\section{Effect of stimulus anticipation during darkness}

The ANOVAs on both the alpha and the beta power did not reveal any significant effect (all of the main effects and interactions $F<1)$. This indicated that oscillations recorded from central electrodes $(\mathrm{C} 3$ and $\mathrm{C} 4)$ during darkness were not modulated by the type of cue provided (about whether the successive stimulation would be 'warm' or 'hot').

\section{Effect of visual context relative to darkness}

Again, the ANOVAs on darkness-rescaled alpha and beta power during vision did not show any significant effect or interaction (refer to Table 1 for detailed statistical results). Therefore, central oscillations at rest were not modulated by the visual context, as compared with darkness (Fig. 4).

\section{Effect of visual plus thermal stimulation relative to vision}

In the alpha band, we found a laterality effect (main effect of hemisphere, Table 2), i.e. alpha power was lower over contralateral 
TABLE 1. Statistical results of the ANOVAs performed on the alpha and beta power during vision, rescaled for darkness time window (Experiment 2)

\begin{tabular}{|c|c|c|c|c|c|}
\hline \multirow[b]{2}{*}{ Source } & \multirow[b]{2}{*}{ df } & \multicolumn{2}{|c|}{ Alpha band } & \multicolumn{2}{|c|}{ Beta band } \\
\hline & & $F$ & Sig. & $F$ & Sig. \\
\hline Hemisphere & 1,10 & 0.43 & 0.526 & 0.37 & 0.555 \\
\hline Context & 1,10 & 3.07 & 0.110 & 0.00 & 0.949 \\
\hline Intensity & 1,10 & 0.01 & 0.908 & & \\
\hline Hemisphere * Context & 1,10 & 0.13 & 0.724 & 0.07 & 0.796 \\
\hline Hemisphere $*$ Intensity & 1,10 & 0.73 & 0.414 & 1.97 & 0.191 \\
\hline Context $*$ Intensity & 1,10 & 0.41 & 0.536 & 2.04 & 0.183 \\
\hline $\begin{array}{l}\text { Hemisphere * } \\
\text { Context * Intensity }\end{array}$ & 1,10 & 0.09 & 0.771 & 0.09 & 0.765 \\
\hline
\end{tabular}

(i.e. C4; mean $\pm \mathrm{SEM},-0.12 \pm 0.24 \mathrm{~dB}$ ) than ipsilateral (i.e. C3; mean $\pm \mathrm{SEM}, 0.67 \pm 0.17 \mathrm{~dB}$ ) sensorimotor areas. No other effect or interaction was significant (Table 2).

In the beta band, there was a similar laterality effect, and a main effect of the visual context (Table 2). There was a significant interaction between the stimulus intensity and the visual context $\left(F_{1,10}=5.16, P=0.047\right)$; the increase in beta oscillations during vision of the body in comparison to the object (Figs 5 and 6) was significant only for noxious stimuli (mean difference \pm SEM, $0.66 \pm 0.20 \mathrm{~dB} ; P=0.007$ ), and not for innocuous warmth (mean difference \pm SEM, $0.18 \pm 0.13 \mathrm{~dB} ; P=0.208$ ).

The interaction hemisphere by intensity was also significant $\left(F_{1,10}=7.18, P=0.023\right)$; follow-up tests showed that there was no significant laterality difference for innocuous warm stimuli $\left(t_{10}=1.61, P=0.137\right)$, whereas there was a significant difference for noxious stimuli $\left(t_{10}=4.53, P=0.001\right)$. In particular, beta oscillations induced by noxious stimulation were reduced over the contralateral sensorimotor cortex $(\mathrm{C} 4:$ mean \pm SEM, $0.04 \pm 0.11 \mathrm{~dB})$, in comparison to the ipsilateral sensorimotor cortex (C3: mean \pm SEM, $0.57 \pm 0.17 \mathrm{~dB}$; see Fig. 6).
TABLE 2. Statistical results of the ANOVAs performed on the alpha and beta power during visual-thermal stimulation, rescaled for the unimodal visual time window (Experiment 2) (Significant values are highlighted in bold)

\begin{tabular}{|c|c|c|c|c|c|}
\hline \multirow[b]{2}{*}{ Source } & \multirow[b]{2}{*}{ df } & \multicolumn{2}{|c|}{ Alpha band } & \multicolumn{2}{|c|}{ Beta band } \\
\hline & & $F$ & Sig. & $F$ & Sig. \\
\hline Hemisphere & 1,10 & 9.97 & 0.010 & 15.34 & 0.003 \\
\hline Context & 1,10 & 0.81 & 0.388 & 10.12 & 0.010 \\
\hline Intensity & 1,10 & 3.77 & 0.081 & 0.96 & 0.350 \\
\hline Hemisphere $*$ Context & 1,10 & 0.56 & 0.473 & 0.69 & 0.427 \\
\hline Hemisphere $*$ Intensity & 1,10 & 0.08 & 0.789 & 7.18 & $\mathbf{0 . 0 2 3}$ \\
\hline Context $*$ Intensity & 1,10 & 0.25 & 0.629 & 5.16 & 0.047 \\
\hline Hemisphere $*$ Context $*$ Intensity & 1,10 & 0.83 & 0.383 & 1.20 & 0.299 \\
\hline
\end{tabular}

The three-way interaction was not significant (Table 2), suggesting that the effects of the visual context on beta oscillations induced by noxious heat were similar for the ipsilateral and contralateral sensorimotor cortices. However, a paired $t$-test on the beta power induced by noxious stimuli in the hand-view condition revealed a laterality difference $\left(t_{10}=2.67, P=0.024\right)$, with reduced power over the contralateral sensorimoror cortex (C4; Figs 5 and 6).

\section{Discussion}

In summary, Experiment 2 revealed that viewing the hand modulated beta sensorimotor oscillations induced by noxious thermal stimuli, as compared with viewing the object. This modulation consisted of a selective enhancement of beta oscillations induced by noxious stimulation, in both the ipsilateral and contralateral sensorimotor cortices. This result suggests that viewing one's own body modulates cortical pain processing.

The block design of the present experiment allowed differentiation between the effects of the visual context and of pain expectation, which could not be separated in Experiment 1. Knowing in
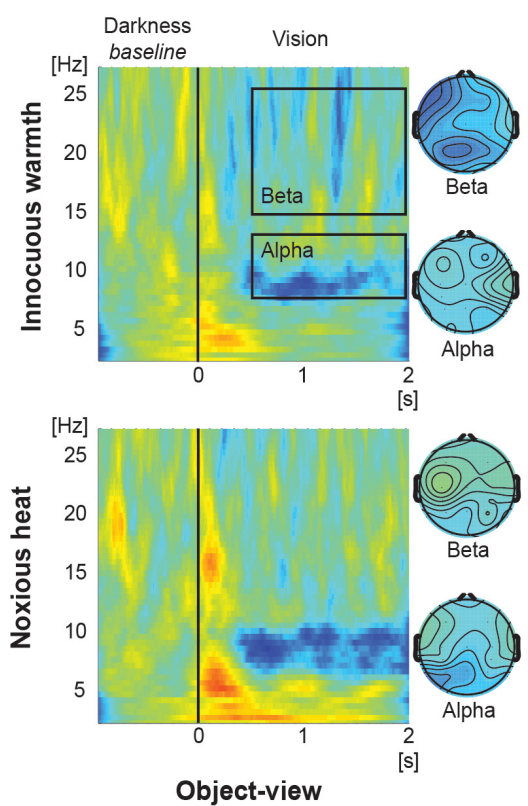

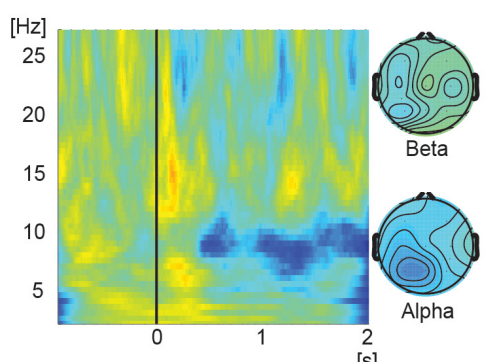

[s]

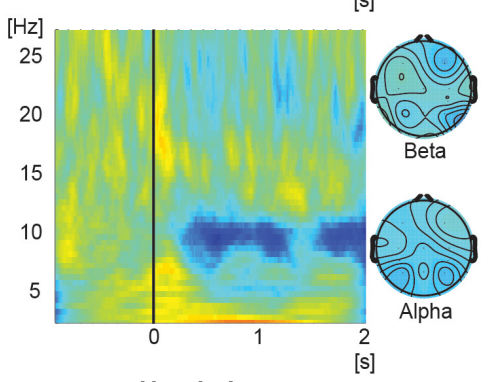

Hand-view

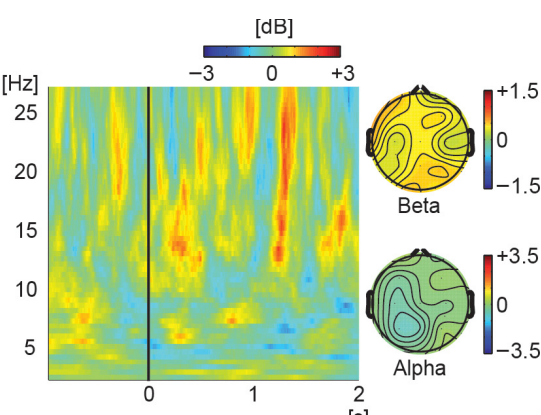

[s]

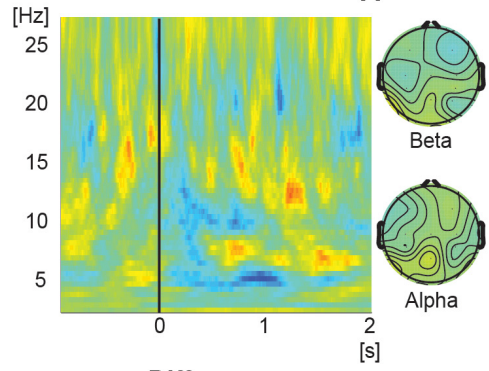

Difference

[hand - object]

FIG. 4. Experiment 2. Grand mean time-frequency representations of EEG spectral power over C4. Responses rescaled for the darkness baseline were averaged across 11 subjects. Topographical maps represent average oscillatory power in the alpha (8-13 Hz) and beta (15-25 Hz) bands during the highlighted section of the visual phase before thermal stimulation. 

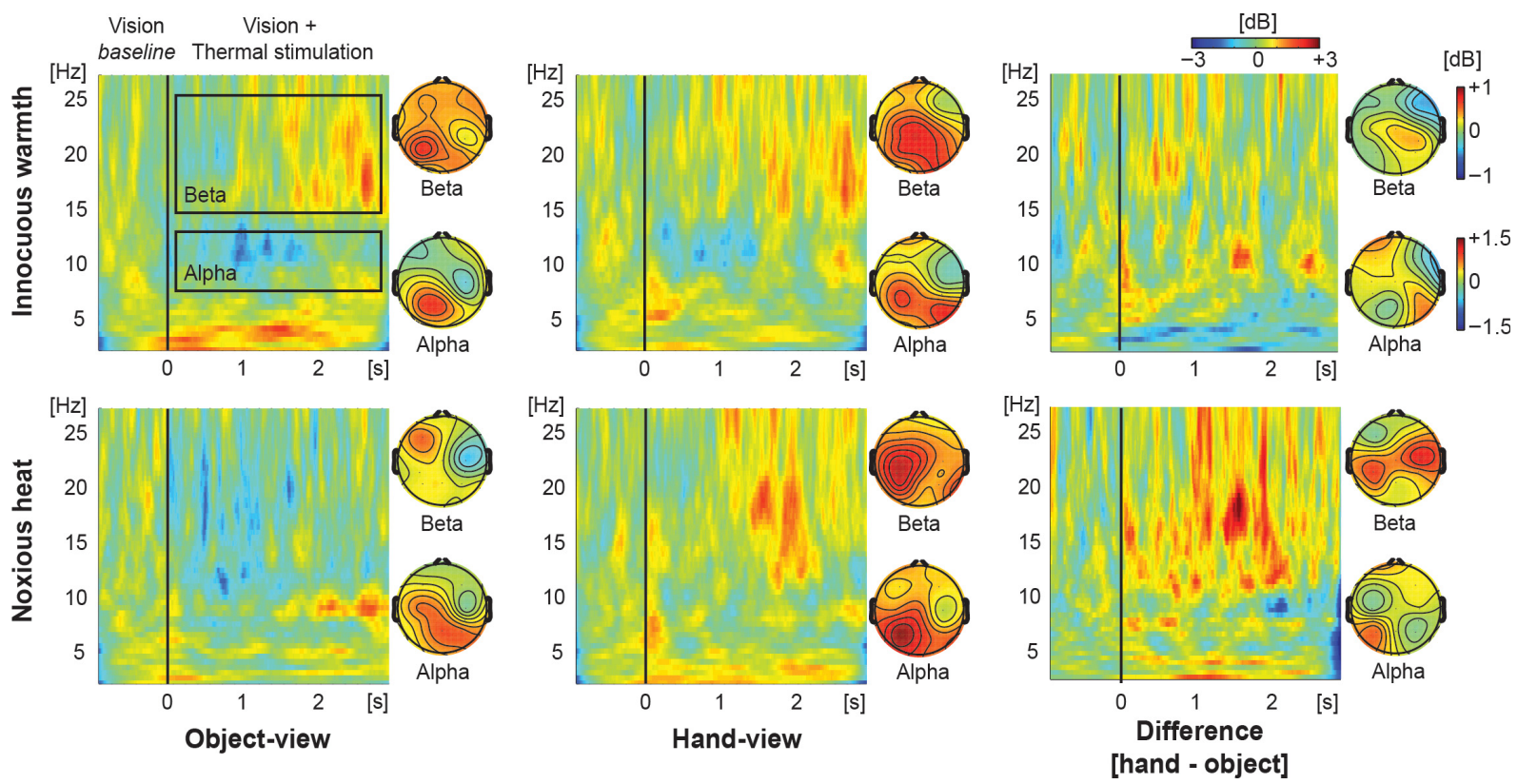

FIG. 5. Experiment 2. Grand mean time-frequency representations of EEG spectral power at electrode C4. Responses rescaled using a baseline period taken from the visual phase were averaged across 11 subjects. Topographical maps represent average oscillatory power during vision plus thermal stimulation in the alpha $(8-13 \mathrm{~Hz})$ and beta $(15-25 \mathrm{~Hz})$ bands.

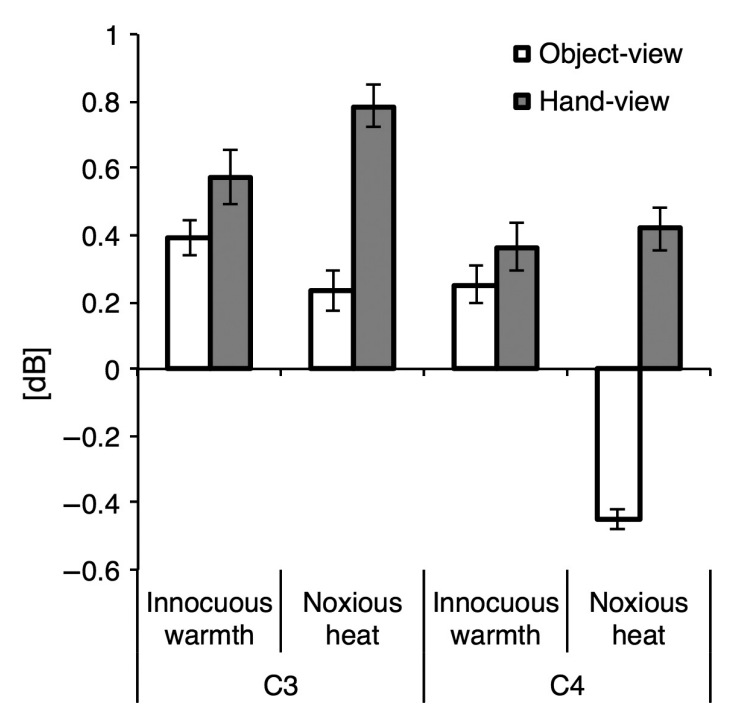

FIG. 6. Experiment 2. Extracted beta power from electrodes C3 (ipsilateral to stimulation) and C4 (contralateral to stimulation) induced by thermal stimulation. Responses rescaled for the visual baseline were averaged across 11 subjects. Bars represent \pm 1 SE.

advance the type of stimulation to come (warm or hot) did not influence EEG oscillations at rest during darkness, suggesting that the role of pain expectation in the EEG activity during our paradigm might be limited. Furthermore, sensorimotor oscillations were not influenced by the visual context during the baseline period (i.e. before any thermal stimulus), as compared with darkness.

\section{General discussion}

The two experiments yielded convergent results regarding the effect of the visual context of viewing one's own body on perceived pain intensity and on sensory EEG rhythms. In both experiments, the perceived intensity of noxious heat stimuli, but not of innocuous warm stimuli, was reduced when viewing one's own body, as compared with viewing an object. Noxious stimuli induced desynchronization of cortical oscillatory activity in the beta band. This pain-induced beta suppression was consistently reduced during vision of the hand, as compared with vision of the object. In other words, the visual context modulated thermally-induced EEG oscillations bilaterally over the somatosensory cortex, resulting in increased beta power for viewing the hand vs. the object. Taken together, these results allow us to understand the brain dynamics underlying the interactions between visual body representation and nociception.

\section{Nociceptive processing}

We compared the effects of noxious heat and innocuous warmth on alpha and beta oscillations. In both experiments, we found that noxious stimuli decreased beta power, as compared with innocuous warmth. The beta power decrease was largely bilateral (Experiment 1) for stimuli of slowly-increasing temperature (12 s long), whereas the effect was predominantly contralateral for shorter stimuli $(3 \mathrm{~s}$ long) maintained at constant temperature (Experiment 2). In Experiment 1 , the decrease in EEG power induced by noxious stimuli also extended to the alpha band.

We interpret these as a relative reduction of beta rhythms linked to nociceptive processing, in line with previous findings (Crone et al., 1998; Mouraux et al., 2003; Raij et al., 2004; Ploner et al., 2006b; Hauck et al., 2007; Stancak et al., 2007; Senkowski et al., 2011). Further, pain-induced oscillatory power correlates negatively with the excitability of the somatosensory cortex (Ploner et al., 2006a). Therefore, oscillatory beta power reduction induced by noxious stimulation presumably reflects the stronger activation of somatosensory areas with more intense thermal stimulation.

\section{Visual modulation of nociceptive processing}

Viewing the hand reduced the perceived intensity of noxious heat, compared with viewing the neutral object. This finding extends 
reports of visually-induced analgesia reported previously (Longo et al., 2009, 2012; Mancini et al., 2011). In contrast, intensity ratings for innocuous warm stimuli were not modulated by the visual context, suggesting that the analgesic effect of vision is due to a specific interaction with nociceptive processing, rather than a general biasing effect on all sensory magnitudes.

Moreover, in both experiments, the beta suppression induced by noxious stimuli was consistently reduced during vision of the hand, as compared with vision of the object. This effect was found bilaterally, and was most prominent at central electrodes. Importantly, no modulation in response to innocuous warmth was observed (Experiment 2). Therefore, vision of the body reduces pain ratings, and enhances sensorimotor, beta oscillations that are induced by noxious stimulation of the viewed body part.

In Experiment 1, but not in Experiment 2, we also found modulations in the alpha band, possibly caused by the unpredictable eventrelated design. When the occurrence of pain was unpredictable (Experiment 1), we observed modulations of alpha oscillations both during the baseline period of vision of the hand (decrease of absolute alpha power) and during thermal stimulation (increase of relative alpha power). When pain was predictable (Experiment 2), no alpha decrease was observed. Therefore, the alpha changes in Experiment 1 might reflect non-specific effects of pain expectation. Indeed, alpha EEG oscillations are known to be suppressed by pain expectation (Babiloni et al., 2008), and more generally by factors such as attention (Ray \& Cole, 1985; Jones et al., 2010) and stimulus anticipation (Bastiaansen \& Hagoort, 2003; Rohenkohl \& Nobre, 2011).

We suggest that it is important to consider first the general relation between EEG oscillatory power and cortical activation, and next how our factors of thermal stimulation and visual context may thus influence cortical activation.

Regarding the first point, a relative increase in stimulus-induced oscillatory power has previously been interpreted as reduced (Pfurtscheller et al., 1996) or inhibited (Klimesch et al., 2007) cortical activation, although these previous studies focussed on alpharather than beta-band electroencephalography. Moreover, the view that event-related synchronization of sensory rhythms reflects cortical inhibition is supported by the fact that GABA agonists such as benzodiazepines robustly increase beta-band EEG power over the motor cortex (e.g. Jensen et al., 2005; Hall et al., 2010). Finally, neuronal synchronization in the beta band is a known correlate of crossmodal processing, and it has been reported in studies using paradigms of passive stimulation (Senkowski et al., 2008).

Regarding the second point, we show that noxious stimulation produced a decrease in beta-band power, relative to innocuous stimulation. We interpret this as an increased cortical activation in response to noxious stimuli as opposed to warmth. Viewing the hand seems to counteract the decrease in beta power observed in response to noxious stimulation during vision of the object (relative increase in beta-band oscillation). This result is consistent with the view that viewing the body reduces the somatosensory cortical response to nociceptive input, effectively inhibiting neural pain processing. Thus, EEG oscillatory power, although only an indirect measure of nociceptive processing, changes when viewing the body in a way that is consistent with the psychophysical findings of visual analgesia.

Interestingly, vision of the body has indeed been shown to modulate somatosensory intracortical inhibition (Cardini et al., 2011). We speculate that these effects may depend on the representations of the body and peripersonal space formed in extrastriate visual-body areas and maintained by the posterior parietal cortex (e.g. Avillac et al.,
2005; Longo et al., 2012). In a recent transcranial direct stimulation study, we demonstrated that visually-induced analgesia may depend on neural signals from the extrastriate visual cortex; specifically, we found that the analgesic effect of viewing the body was enhanced selectively by anodal/excitatory stimulation of the occipital cortex (Mancini et al., 2012). Parietal multisensory areas might also in turn modulate networks of inhibitory interneurons in early somatosensory areas. This increased somatosensory inhibition might then be responsible for reduced pain levels (Longo et al., 2012). Viewing the body also influences other somatosensory systems; for touch, it has been demonstrated to have a different effect on spatial acuity, enhancing tactile two-point discrimination (Kennett et al., 2001).

The finding that viewing the body reduces acute pain may seem counterintuitive, given that vision of threatening stimuli, such as needles, increases pain (Van Damme et al., 2010; Hofle et al., 2012). However, in our study, the participants' attention was not explicitly directed towards or away from noxious stimulation. We did, however, manipulate the subjective certainty about the thermal stimulation. We observed similar modulations of nociceptive processing by vision of the body, both when participants could fully predict that the stimulation would be noxious (Experiment 2), and when they could not (Experiment 1). Conversely, facilitation of pain by attention to a potentially threatening stimulus is not elicited only by vision (Hofle et al., 2012), but also by almost any other sensory input that has previously been associated with pain (Koyama et al., 2005; Atlas et al., 2010). Here we have focused on a more specific, but less well-known, form of pain modulation - visual analgesia from the visual context of viewing the body. The present study indicates that pain perception and sensorimotor oscillations induced by noxious thermal stimulation are modulated by the visual context of viewing one's own body.

\section{Acknowledgement}

F.M. was supported by a doctoral program of the University of Milano-Bicocca. G.V. was supported by PRIN and FAR grants. P.H. was supported by a Research Fellowship from the Leverhulme Trust, and by EU FP7 project VERE.

\section{Abbreviation}

EEG, electroencephalographic.

\section{References}

Atlas, L.Y., Bolger, N., Lindquist, M.A. \& Wager, T.D. (2010) Brain mediators of predictive cue effects on perceived pain. J. Neurosci., 30, 12964 12977.

Avillac, M., Deneve, S., Olivier, E., Pouget, A. \& Duhamel, J.R. (2005) Reference frames for representing visual and tactile locations in parietal cortex. Nat. Neurosci., 8, 941-949.

Babiloni, C., Capotosto, P., Brancucci, A., Del Percio, C., Petrini, L., Buttiglione, M., Cibelli, G., Romani, G.L., Rossini, P.M. \& Arendt-Nielsen, L. (2008) Cortical alpha rhythms are related to the anticipation of sensorimotor interaction between painful stimuli and movements: a high-resolution EEG study. J. Pain, 9, 902-911.

Bastiaansen, M. \& Hagoort, P. (2003) Event-induced theta responses as a window on the dynamics of memory. Cortex, 39, 967-992.

Cardini, F., Longo, M.R. \& Haggard, P. (2011) Vision of the body modulates somatosensory intracortical inhibition. Cereb. Cortex, 21, 2014-2022.

Crone, N.E., Miglioretti, D.L., Gordon, B., Sieracki, J.M., Wilson, M.T., Uematsu, S. \& Lesser, R.P. (1998) Functional mapping of human sensorimotor cortex with electrocorticographic spectral analysis. I. Alpha and beta event-related desynchronization. Brain, 121, 2271-2299.

Delorme, A. \& Makeig, S. (2004) EEGLAB: an open source toolbox for analysis of single-trial EEG dynamics including independent component analysis. J. Neurosci. Methods, 134, 9-21. 
Downing, P.E., Jiang, Y., Shuman, M. \& Kanwisher, N. (2001) A cortical area selective for visual processing of the human body. Science, 293, 2470 -2473 .

Gybels, J., Handwerker, H.O. \& Van Hees, J. (1979) A comparison between the discharges of human nociceptive nerve fibres and the subject's ratings of his sensations. J. Physiol., 292, 193-206.

Hall, S.D., Barnes, G.R., Furlong, P.L., Seri, S. \& Hillebrand, A. (2010) Neuronal network pharmacodynamics of GABAergic modulation in the human cortex determined using pharmaco-magnetoencephalography. Hum. Brain Mapp., 31, 581-594.

Hari, R. \& Salmelin, R. (1997) Human cortical oscillations: a neuromagnetic view through the skull. Trends Neurosci., 20, 44-49.

Hauck, M., Lorenz, J. \& Engel, A.K. (2007) Attention to painful stimulation enhances gamma-band activity and synchronization in human sensorimotor cortex. J. Neurosci., 27, 9270-9277.

Hofle, M., Hauck, M., Engel, A.K. \& Senkowski, D. (2012) Viewing a needle pricking a hand that you perceive as yours enhances unpleasantness of pain. Pain, 153, 1074-1081.

Jensen, O., Goel, P., Kopell, N., Pohja, M., Hari, R. \& Ermentrout, B. (2005) On the human sensorimotor-cortex beta rhythm: sources and modeling. Neuroimage, 26, 347-355.

Jones, S.R., Kerr, C.E., Wan, Q., Pritchett, D.L., Hamalainen, M. \& Moore, C.I. (2010) Cued spatial attention drives functionally relevant modulation of the mu rhythm in primary somatosensory cortex. J. Neurosci., 30, $13760-13765$.

Jung, T.P., Makeig, S., Humphries, C., Lee, T.W., McKeown, M.J., Iragui, V. \& Sejnowski, T.J. (2000) Removing electroencephalographic artifacts by blind source separation. Psychophysiology, 37, 163-178.

Kennett, S., Taylor-Clarke, M. \& Haggard, P. (2001) Noninformative vision improves the spatial resolution of touch in humans. Curr. Biol., 11, 1188-1191.

Klimesch, W., Sauseng, P. \& Hanslmayr, S. (2007) EEG alpha oscillations: the inhibition-timing hypothesis. Brain Res. Rev., 53, 63-88.

Koyama, T., McHaffie, J.G., Laurienti, P.J. \& Coghill, R.C. (2005) The subjective experience of pain: where expectations become reality. Proc. Natl Acad. Sci. USA, 102, 12950-12955.

Legrain, V., Mancini, F., Sambo, C.F., Torta, D.M., Ronga, I. \& Valentini, E. (2012) Cognitive aspects of nociception and pain. Bridging neurophysiology and neuropsychology. Neurophysiol. Clin., 42, 325-336.

Longo, M.R., Cardozo, S. \& Haggard, P. (2008) Visual enhancement of touch and the bodily self. Conscious. Cogn., 17, 1181-1191.

Longo, M.R., Betti, V., Aglioti, S.M. \& Haggard, P. (2009) Visually induced analgesia: seeing the body reduces pain. J. Neurosci., 29, 12125-12130.

Longo, M.R., Iannetti, G.D., Mancini, F., Driver, J. \& Haggard, P. (2012) Linking pain and the body: neural correlates of visually induced analgesia. J. Neurosci., 32, 2601-2607.
Mancini, F., Longo, M.R., Kammers, M.P. \& Haggard, P. (2011) Visual distortion of body size modulates pain perception. Psychol. Sci., 22, 325330 .

Mancini, F., Bolognini, N., Haggard, P. \& Vallar, G. (2012) tDCS modulation of visually-induced analgesia. J. Cogn. Neurosci., 24, 2419-2427.

Mouraux, A., Guerit, J.M. \& Plaghki, L. (2003) Non-phase locked electroencephalogram (EEG) responses to $\mathrm{CO} 2$ laser skin stimulations may reflect central interactions between A partial partial differential- and C-fibre afferent volleys. Clin. Neurophysiol., 114, 710-722.

Neuper, C., Wortz, M. \& Pfurtscheller, G. (2006) ERD/ERS patterns reflecting sensorimotor activation and deactivation. Prog. Brain Res., 159, $211-$ 222.

Pfurtscheller, G., Stancak, A. Jr. \& Neuper, C. (1996) Event-related synchronization (ERS) in the alpha band - an electrophysiological correlate of cortical idling: a review. Int. J. Psychophysiol., 24, 39-46.

Ploner, M., Gross, J., Timmermann, L., Pollok, B. \& Schnitzler, A. (2006a) Oscillatory activity reflects the excitability of the human somatosensory system. Neuroimage, 32, 1231-1236.

Ploner, M., Gross, J., Timmermann, L., Pollok, B. \& Schnitzler, A. (2006b) Pain suppresses spontaneous brain rhythms. Cereb. Cortex, 16, 537-540.

Raij, T.T., Forss, N., Stancak, A. \& Hari, R. (2004) Modulation of motorcortex oscillatory activity by painful Adelta- and C-fiber stimuli. Neuroimage, 23, 569-573.

Ramachandran, V.S., Rogers-Ramachandran, D. \& Cobb, S. (1995) Touching the phantom limb. Nature, 377, 489-490.

Ray, W.J. \& Cole, H.W. (1985) EEG alpha activity reflects attentional demands, and beta activity reflects emotional and cognitive processes. Science, 228, 750-752.

Rohenkohl, G. \& Nobre, A.C. (2011) Alpha oscillations related to anticipatory attention follow temporal expectations. J. Neurosci., 31, 1407614084.

Senkowski, D., Schneider, T.R., Foxe, J.J. \& Engel, A.K. (2008) Crossmodal binding through neural coherence: implications for multisensory processing. Trends Neurosci., 31, 401-409.

Senkowski, D., Kautz, J., Hauck, M., Zimmermann, R. \& Engel, A.K. (2011) Emotional facial expressions modulate pain-induced beta and gamma oscillations in sensorimotor cortex. J. Neurosci., 31, 14542-14550.

Stancak, A., Polacek, H., Vrana, J. \& Mlynar, J. (2007) Cortical oscillatory changes during warming and heating in humans. Neuroscience, 147, 842852.

Van Damme, S., Legrain, V., Vogt, J. \& Crombez, G. (2010) Keeping pain in mind: a motivational account of attention to pain. Neurosci. Biobehav. Rev., 34, 204-213

Wiech, K., Ploner, M. \& Tracey, I. (2008) Neurocognitive aspects of pain perception. Trends Cogn. Sci., 12, 306-313. 